\title{
Bone marrow adiposity and multiple myeloma
}

Emma V. Morris ${ }^{1}$ and Claire M. Edwards $s^{1,2}$

${ }^{1}$ Nuffield Dept. of Surgical Sciences, University of Oxford, Oxford, UK. ${ }^{2}$ Nuffield Dept. of Orthopaedics, Rheumatology and Musculoskeletal Sciences, University of Oxford, Oxford, UK

Corresponding author: Claire Edwards,

Botnar Research Centre, University of Oxford

Old Road

Oxford OX3 7LD

Email: claire.edwards@ndorms.ox.ac.uk 


\begin{abstract}
Multiple Myeloma (MM) is an incurable haematological malignancy and is the second most common blood cancer in adults; it is caused by the clonal expansion of abnormal plasma cells within the bone marrow characterized by osteolytic bone lesions, bone pain, renal disease, and immunodeficiency. MM cells infiltrate the bone marrow where they hijack the microenvironment to sustain growth and survival. The contribution to this process by resident bone cells is well defined. However, the role of bone marrow adipocytes is less clear. As one of the most abundant cell types in the bone marrow these cells are surprisingly understudied. However, in the last few decades they have been recognised as having endocrine function. Adipocytes are metabolically active cells that secrete adipokines, growth factors, and inflammatory mediators, they influence the behaviour and function of neighbouring cells; and have the potential to dysregulate normal bone homeostasis. This review discusses how adipocytes contribute to the metastatic niche in multiple myeloma and cancers that metastasise to the bone and how these new discoveries may contribute to further understanding the mechanisms driving the devastating bone disease associated with MM.
\end{abstract}




\section{Introduction}

There is evidence to suggest that multiple myeloma ( $\mathrm{MM}$ ) has been present for centuries, with cases documented as far back as A.D. 200 [1]. However, one of the first well-documented case was in 1844 by the physician Samuel Solly. Sarah Newbury was a 39-year-old woman who had first complained of severe back pain 4 years previously. When she died in 1844, her autopsy showed that a number of her bones were thinning and her right radius and ulna, left tibia and fibula, and both femurs were fractured. Solly reported that sections of the bones revealed a 'red grumous matter' (thick and lumpy), ranging from a deep Modena red to a bright scarlet crimson. On examination of this red matter it was documented that most of the nucleated cells had a clear, oval outline and one or, rarely, two bright central nucleoli [2]. This was the first detailed descriptions of myeloma cells in the bone marrow and the resulting devastating destruction of the bones. Another case recorded around the same time was that of Thomas Alexander McBean a well-respected grocer who again presented with bone pain and fractures. After his death in 1846 his autopsy revealed; the 'ribs crumbled under the heel of the scalpel.' They were soft and so brittle that they 'could be easily cut by the knife, and readily broken.' The interior of the ribs was filled with a soft 'gelatiniform substance of a blood-red colour and unctuous feel.' The sternum was soft and fragile and snapped when raised and turned back [3]. The bones were observed to have a similar appearance of those of Sarah Newbury. Another important observation made in the case of McBean was the change in his urine [4]. It was described as opaque, acidic and of high density, this was a reference to the large amounts of paraprotein present in the urine which unbeknown at the time is a common symptom of MM.

Today MM is well recognised as a condition that causes bone pain, pathologic fractures, weakness, anaemia, infection (often pneumococcal), hypercalcemia, spinal cord compression, and renal failure [5]. MM is caused by the clonal expansion of abnormal plasma cells which thrive in the bone promoting debilitating bone destruction by initiating complex interactions with other cells of the bone microenvironment. The bone consists of a number of different cell types, including nerve cells and endothelial cells, cells of hematopoietic origin, such as hematopoietic stem cells, osteoclasts macrophages, and lymphocytes, as well as cells from mesenchymal origin, such as osteoblasts, osteocytes, chondrocytes and adipocytes. In order to maintain healthy bone these cells provide a supportive niche via cellular cross talk. However, in the event of disease such as in the cases of Sarah Newbury and Thomas McBean this protective niche can be commandeered by the cancer cells and utilised to support tumour growth at the expense of bone integrity.

In order to target the bone microenvironment effectively the contribution of each cell type needs to be established. Mundy and colleagues introduced the concept of the "vicious-cycle" which described 
bi-directional interactions of cancer cells and bone cells, resulting in osteolysis and in turn, tumour growth. Cancer cells produce growth factors that activates osteoblasts to secrete receptor activator of nuclear factor kappa-B ligand (RANKL) and downregulate osteoprotegerin (OPG). In turn, this activates osteoclast precursors to increase osteoclast recruitment and formation resulting in the breakdown of the bone matrix and the release of bone-derived growth factors, such as transforming growth factor- $\beta$ and insulin-like growth factor 1 , and raises extracellular calcium concentrations. These growth factors then bind to receptors on the tumour cell surface activating SMAD and MAPK signalling, extracellular calcium binds and activates calcium pumps leading to tumour cell proliferation thereby creating a vicious cycle [6]. This process results in patients with MM having abnormal bone remodelling, where resorption and formation become uncoupled, with an increase in bone resorption and a decrease in bone formation [7]. Myeloma cells are found in close association with sites of active bone resorption suggesting that bone disease is mediated locally [8] agreeing with the theory that tumour and bone cells have a reciprocal relationship resulting in the occurrence of lytic lesions in the bone. Tumour cells are masters of manipulation causing their environment to change to their advantage, bone resorption not only releases growth factors it also creates space for tumour cell establishment. However as with most biological processes it is far more complex than a simple see-saw effect between osteoblasts and osteoclasts. Granted, osteoblasts and osteoclasts are the gate keepers of normal bone degradation and formation. However other resident cells also play a role in maintaining a healthy environment. Mesenchymal stem cells (MSCs) in bone marrow give rise to stromal cells. These stromal cells are known to support the homing, proliferation, differentiation, and survival of hematopoietic cells, however by the same mechanisms stromal cells can also promote cancer cell migration into the bone marrow [9]. Stromal stem cells can differentiate into many different cell types depending on their environmental cues $[10,11]$. One cell type that has gained a lot of attention in the last few decades is one of the most abundant cell types in the marrow; the bone marrow adipocyte. Once thought to be an inert space filling cell it has now been recognised as having important endocrine functions [12] as well as being a key player in bone homeostasis and a potential driver of tumour cell establishment and progression [13-16].

\section{Bone marrow biology}

Within the medullary cavity of the bone there are two distinct types of bone marrow which vary in composition and function, red (hemopoietic, active marrow) and yellow, (fatty, inactive marrow). The red colour is due to the hemoglobulin in the red cells and the yellow colour is produced by carotenoids in the fat cells. Marrow cavities in all the bones of newborn mammals contain hematopoietic red bone marrow. From early postnatal period onwards, the hematopoietic tissue, 
mainly in the bones of the extremities, is gradually replaced by non-hematopoietic MSCs that have started to accumulate lipids, these cells differentiate into mature adipocytes forming fatty yellow adipose tissue, creating a distinct patterning of red vs. yellow marrow in the skeleton. The fact that fatty marrow develops in this conserved, spatial, and temporal manner implies that is has a defined physiological function. By the age of 25 , approximately $70 \%$ of bone volume is occupied by yellow marrow. Although variation exists, by this age the hematopoietically active red marrow is mainly confined to the spine, ribs, sternum, pelvis and skull and to a lesser degree in the proximal metaphyseal regions of the humeri and femora [17], whilst the majority of the long bones contain yellow marrow. However, this is not mutually exclusive as by around 70 years of age even the red marrow within the axial sites contains more than $50 \%$ fat [18]. This observation is supported by longitudinal studies of patients from birth to 90 years old that show after the initial exponential accumulation of fatty marrow, there continues to be a gradual increase in bone marrow adiposity throughout adult life [19]. The exact reasons for this massive conversion from red to yellow marrow is still unclear. It is thought that the amount of red marrow may correlate with the number of healthy proliferating MSCs. As we age our MSC pool capable of supporting haematopoiesis decreases thereby promoting the accumulation of adipose tissue [20]. Adipocytes thereby make up a large component of the bone microenvironment. Is this conversion protective to the skeleton or detrimental? Or is it a simple consequence of old age? Many diseases such as cancer preferentially effect the elderly. As we age our bodies become more susceptible to mutations, DNA damage and dysregulated gene function [21]. Does an increase in adiposity affect disease development and progression and if so are there ways of targeting adipocytes to treat these conditions? These are all important questions currently under investigation by researchers in the field.

\section{Bone marrow adipocytes}

Adipocytes are derived from multipotent MSCs that are capable of differentiating into myocytes, chondrocytes, osteoblasts and adipocytes [11]. It is postulated that downstream of these multipotent progenitors, bipotent osteoblast-adipocytes progenitors form an intermediate in this process [22]. Commitment to either these two cell lineages is stimulated by the presence of adipogenic vs. osteogenic factors within the bone microenvironment that activate their respective transcriptional programs $[23,24]$. In childhood and early adulthood commitment appears to support osteoblastogenesis however as we age that commitment decision changes in favour of adipogenesis. This phenomenon can also be affect by lifestyle such as a lack of exercise and/or a high fat diet causing obesity which also increases marrow adiposity [25]. Whether this obesity-induced increase mimics aging or whether it promotes further changes to the bone microenvironment is not yet clear. Marrow adipocytes are smaller than their subcutaneous and visceral counterparts, although due to 
enhanced triacylglycerol synthesis they are just as efficient at storing triacylglycerol during energy excess and releasing fatty acids in times of energy demand. As with all adipocytes of the body their main function is to store energy, however, they are also responsible for secreting an array of signalling molecules known as adipokines. These adipokines function as classic circulating hormones to communicate with other organs such as the brain, liver, muscle, the immune system, and adipose tissue itself. Two of the most abundant and highly studied adipokines are leptin and adiponectin, which regulate calorie uptake and insulin sensitivity, respectively. Adipocytes also secrete several cytokines such as IL-6, IL-1 $\beta$, and TNF- $\alpha$ [26] which play a role in regulating adipose tissue metabolism [27]. Adipose tissue is now recognised as the largest endocrine organ of the body, inputting signals into numerous physiological processes. Bone marrow adipose tissue (BMAT) appears to be sensitive to metabolic changes in bone, such as increasing in size and number in response to a high fat diet. Interestingly an increase is also observed upon starvation. Which is converse to the response seen in white and brown adipose depots that rapidly decrease in response to starvation [28]. To add another level of complexity there are two types of bone marrow adipose tissue, known as regulated (rBMAT) and constitutive (cBMAT). It is thought that cBMAT accumulates with age, and resembles the tightly packed structure of white adipose tissue. It is located in the less metabolically active sites of the bone such as the ankle. Whereas rBMAT is made up of more dispersed cells found scattered among the hematopoietic cells near the growth plate and trabecular in sites of active bone remodelling. rBMAT is thought to be the main responder to changes in metabolic demands increasing and decreasing in response to energy needs [29]. The location of these cells suggests that rBMAT could play a role in the bone remodelling process. This is a theory that warrants closer examination. Cancer cells that reside in the bone cause devastating destruction, could the adipokines secreted from these adipocytes promote cancer establishment and progression? If rBMAT is not a contributing factor to bone turn over then why is it found predominantly in the metabolically active sites of the bone? It is not just mature adipocytes that need to be considered. Pre-adipocytes are also metabolically active cells which are known to express RANKL [30], since during disease establishment the number of pre-adipocytes are increased this may also contribute to bone disease and further drive the vicious cycle. These are questions that are starting to be addressed in the field and hopefully understanding marrow adiposity more thoroughly will allow for more effective treatments for patients who suffer with associated bone diseases. Particularly for patients who suffer from cancers which metastasise to the bone, or patients that suffer from cancers such as MM that are derived from within the bone.

\section{Cancer associated bone disease}


The bone marrow is a favoured site for several cancers, including multiple myeloma, and metastasis of breast and prostate cancer. Around $70-80 \%$ of patients with advanced breast or prostate cancer suffer from bone metastasis. This raises the question as to why these cancer cells preferentially home to the bone. Metastasis is a highly inefficient process with $<0.1 \%$ of disseminating cells surviving to form secondary lesions [31] and even with these odds we see a $70 \%$ success rate. It has long been recognized that each tumour type has a distinct pattern of dissemination. This would infer that for breast and prostate cancer cells the bone is an environment that lends itself to adaptation. Both these cell types have been shown to participate in the "vicious cycle" promoting bone destruction, thereby promoting the release of vital growth factors. However, is this enough for these cells to thrive and cause so much destruction or do they need to interact with other cells in the bone microenvironment? Over the past decade there has been a number of publications that have implicated rBMAT as a driver of prostate cancer bone metastasis. Adipocytes that reside in the red marrow promote homing to the bone, and fuel progression by the translocation of lipid from the adipocyte to the cancer cell via the upregulation of the fatty acid chaperone FABP4 $[13,32,33]$. Using a mouse model of diet-induced marrow adiposity Herroon and colleagues demonstrated that alongside the upregulation of FABP4, interleukin $1 \beta$ and its target gene, oxidative stress protein, heme oxygenase 1 (HMOX-1) was also upregulated in prostate cancer cells that were in direct contact with bone marrow adipocytes, supporting the fact that tumour cells utilize marrow adipocyte-supplied lipids to thrive in skeletal sites. [13]. The contribution to the associated bone disease is thought to be driven in part by the upregulation of the chemokines, CXCL1 and CXCL2. Adipocytes are a major source of CXCL1 and CXCL2, which is upregulated by adipocyte-tumour interactions. These chemokines activate osteoclastogenesis thus promoting tumour induced osteolysis [34]. These findings support the notion that rBMAT may play an active part in normal bone turnover, and that this process is modified by the presence of cancer cells. Breast cancer cells have also been shown to be recruited to human bone tissue-conditioned medium by adipocyte derived factors. Templeton and colleagues demonstrated that leptin and IL-1 $\beta$ caused colonization of the bone marrow adipose tissue compartment of cultured bone tissue fragments, suggesting that they play a functional role in the breast cancer metastatic niche [35].

\section{Regeneration}

As well as adipocytes being involved in promoting cancer progression they have also been identified as playing a protective role in restoring normal haematopoiesis after chemotherapy treatment. Adipocytes secrete the cytokine stem cell factor (scf) which promotes the survival, proliferation, and differentiation of hematopoietic stem cells and progenitor cells. Thus, adipocytes promote the regeneration of hematopoietic stem cells and haematopoiesis after irradiation therapy or 5- 
fluorouracil (5-FU) treatment [36]. Patients with acute myeloid leukaemia suffer from fatal infections and anaemia due to haematopoietic failure. These patients have a depletion of rBMAT in the marrow. This was first thought to be due to simply out crowding by the tumour cells. However, it has now come to light that leukaemia cells utilise adipocytes via lipids translocation in a similar manner to prostate cancer cells, thereby, depleting the adipocyte pool. The consequence of this is a decrease in proliferation of the hematopoietic stem cells resulting in the prevention of normal blood production. [37]. These findings suggest that following chemotherapy, stimulation of the adipocyte niche may be a potential therapeutic strategy, highlighting the importance of marrow adipose tissue. Although adipocytes clearly promote cancer cell survival they also play a positive role during therapy. Targeting these cells could be challenging, suppressing the functions and secretions that driver cancer progression and abnormal bone turnover, whilst keeping a healthy balance for future regeneration.

\section{Multiple Myeloma}

$\mathrm{MM}$ is most prevalent in people over the age of 55 years of age with incidence in the UK peaking in the 85 - 89 age range [38]. If adiposity continues to accumulate over time by the age of $55-90$ years old the proportion of yellow marrow vs. red marrow is considerable. Could this level of adiposity be creating a permissive environment for myeloma cells to establish and thrive? Myeloma is known to preferentially home to the spine, pelvis, ribs, skull and metaphyses of long bones, intriguingly with distribution similar to that of red bone marrow [39]. In 2016 the International Agency for Research on Cancer (IARC) listed myeloma as one of 13 types of cancer that was positively associated with obesity [40]. More work needs to be done to understand the details of this association. Is it an accumulation of white adipose tissue or is it a consequence of an increase in marrow adiposity caused by obesity that is promoting the disease? Unlike most other conditions myeloma is nearly always preceded by a condition known as Monoclonal Gammopathy of Undetermined Significance (MGUS). Obesity is positively correlated with progression from MGUS to myeloma. In fact a study carried out by Chang and colleagues showed that obese patients are $20 \%$ more likely to progress to full blown disease. Therefore, by understanding the disease further and the interactions that drive the progression from MGUS to myeloma there is a window of opportunity to advice MGUS suffers to modify their lifestyle thus modifying their risk of progression. MGUS patients present with abnormal paraprotein in their blood and urine just as in the case of McBean. However, the difference being that they do not suffer with any of the other complications such as the associated bone disease. Living with MGUS has often been described as living like a ticking time bomb with no idea of when, if indeed at all, the condition will progress. Could it be that a normal proportion of marrow adiposity is 
healthy and protective, however, if that balance is lost and extra BMAT accumulates it could become a fuel for disease initiation and progression.

\section{Myeloma bone disease}

MM-induced bone disease occurs as myeloma cells colonise the bone marrow, upon engraftment they initialise the steps of the vicious cycle accelerating osteoclastogenesis through the expression of molecules including MIP-1alpha RANKL, MMP-13 and Decoy receptor 3 (DcR3) [41-43]. In addition, myeloma cells inhibit osteoblastogenesis through expression of Dickkopf-1 (DKK-1) [44] and induction of increased sclerostin levels from neighbouring osteocytes [45]. This results in the disruption of normal bone remodelling skewing the balance towards increased osteolytic processes. These pathological processes are well defined in terms of the link between resident bone cells and invading MM cells. However, as with other metastatic cancers there is now emerging evidence to suggest that $\mathrm{MM}$ cells are fuelled and driven by more than bone cell interaction. Both preadipocytes and mature adipocytes have been shown to promote MM migration, proliferation and survival. MM cells are recruited by adipocytes to the bone marrow via the expression of chemoattractant molecules such as MCP-1 and SDF-1 $\alpha$ [46]. On establishment in the bone marrow MM cells thrive by utilising growth factors secreted by cells of the bone microenvironment such as IL-6, TNF- $\alpha$, IGF-1 and HGF [47-50] to promote adhesion, proliferation and overall survival. Adhesion of $\mathrm{MM}$ to adipocytes appears to offer a level of protection against apoptosis having a detrimental effect on the efficacy of chemotherapy [26]. Adipocytes also secrete a plethora of adipokines some of which are known to be dysregulated in MM such as leptin, adipsin, adiponectin visfatin and resistin. Leptin is a well-known adipokine responsibly for satiety, which has been found to be elevated in MM patients, with levels reducing in response to disease treatment [51]. Leptin and adipsin play a role in chemo-resistance by inducing autophagy via Jak/Stat3 signalling, thereby reducing chemotherapy-induced apoptosis [52]. Adiponectin is known as a 'good' adipokine exerting anti-tumour effects by decreasing cellular proliferation and increasing apoptosis of myeloma cells via the activation of AMPK and MAPK [53]. Adiponectin levels are found to be lowered in the serum of MM patients [54]. Visfatin has been shown to be tumour promoting; myeloma cells treated with the PBEF1 inhibitor APO866 had a reduction in cellular proliferation coupled by an induction of apoptosis [55]. Resistin has been found to be a double-edged sword with low levels of resistin being associated with risk [54], and high levels are linked to the promotion of drug resistance, abrogating chemotherapy-induced apoptosis in myeloma cells via the inhibition of chemotherapy-induced caspase cleavage [56]. These findings may suggest that resistin plays a different role in different stages of the disease. Examples like this one highlight how heterogeneous, and constantly evolving these tumour cells are. However, for many of these adipokines they could 
be potentially promising targets for future treatment strategies. As well as utilising adipokines MM cells also respond to locally released growth factors to promote proliferation however it is not yet clear whether they also utilise lipid translocation in a comparable manner to prostate, leukaemia and ovarian cancer cells [57] to also fuel their progression.

Since adipocytes and osteoblasts are derived from a common progenitor any shift in the direction of increased adipogenesis would have a negative effect on osteoblastogenesis. This phenomenon is observed in post-menopausal women [58] degenerative bone disorders such as osteoporosis [59, 60] and obesity [61] and is associated with poor bone quality. MM progression is positively correlated with obesity suggesting that an increase in adiposity creates a more favourable environment. This could be due to increased FFA release as a consequence of elevated adipocyte numbers, the changes in adipokine secretion that accompanies obesity or the imbalance of resident cells in the microenvironment. Moreover, it could be a combination of any or all of these factors. The changes in circulating factors secreted by both pre-adipocytes and mature adipocytes may have an overarching effect on the microenvironment itself. Obesity causes a low level of chronic inflammation recruiting macrophages into the marrow, macrophages are a major source of TNF- $\alpha$, TNF- $\alpha$ activates RANKL expression in the adipocytes as well as osteoblasts which in turn activates osteoclast differentiation [62]. Therefore an increase in adipocyte number could potentially result in enhanced abnormal bone remodelling and thus further promote bone disease. These changes consequentially support MM cell growth allowing them to thrive and utilise the adipocytes as an energy source. It is interesting that MM is a cancer of multiple sites but has never been reported in WAT. This suggests either that the differences in BMAT and WAT are subtle enough to have completely different effects on MM cells or that an environment that consists of adipocytes alone is not a favourable one, and that it is the additive effect of an increase in adiposity alongside a dysregulation of bone turnover that drives disease. It could be described that the bone destruction is generated by a machine composed of bone cells, driven by MM cells and fuelled by adipocytes. One question that remains unanswered is whether MM cells themselves induce an increase in adiposity at any stage of disease, thereby causing favourable adaptation from the outset. However, what is clear is that the combination of an increase created in BMAT and an uncoupling of normal bone degradation and formation promotes $\mathrm{MM}$ and its associated bone disease.

\section{Conclusion}

We have learnt a great deal about the bone microenvironment and its role in disease since both Sarah Newbury's and Alexander McBean's conditions were first documented. They were treated with old-fashioned tonics, infusions of orange peel and rhubarb pills. Today patients are treated with 
more strategic therapies such as dexamethasone, bortezomib, carfilzomib and ixazomib which specifically target the $\mathrm{MM}$ cells, often in combination with antiresorptive agents such as Bisphosphonates that target the bone disease. However, even with modern treatments MM remains an incurable disease. This suggests that a piece of the puzzle is missing, why are these therapies not enough, what does the bone microenvironment offer that is protecting these cells, and how can it be targeted? With this in mind a more concerted approach is needed. The influence of other resident cells needs to be considered. There is mounting evidence that increased adiposity is tumour promoting and so using therapies that cause apoptosis to not only MM cells but other cells in the marrow could create more space for further BMAT expansion which may have a negative effect on long-term treatment outcomes. One signalling pathway that has gained a lot of attention as a potential therapeutic target is the Wnt signalling pathway. Wnt signalling pushes MSCs to preferentially differentiate down the osteoblastic lineage, thereby inhibiting adipogenesis. DKK1 and sclerostin are both Wnt inhibitors and are found to be elevated in MM patients. Anti-DKK1 antibodies and anti-sclerostin antibodies may be potential therapeutic candidates for future therapies as these act in two ways; increasing osteoblastogenesis which would have a positive effect on bone disease whilst inhibiting adipogenesis $[63,64]$. These treatments need to be validated in clinical trials so the verdict is still out as to whether they are effective treatment options. It may be that developing drugs to target the bone marrow microenvironment in the pursuit of a cure for $\mathrm{MM}$ is still only an idea. However, in the management and monitoring of MGUS this is a different story. There is enough evidence to suggest that MGUS patients should be advised to consider their weight as a factor for progression and that it should be standard practice to advise these patients that lifestyle could be the ultimate lifesaver. This is an exciting time in the marrow adiposity field with momentum building as more research groups take up the challenge of understanding more about how these cells and their contribute to both bone health and disease.

\section{Acknowledgements}

This work was supported by Bloodwise, a Marie Curie Career Integration Grant from the European Union Seventh Framework Programme and the National Institute for Health Research (NIHR) Oxford Biomedical Research Centre (BRC). The views expressed are those of the author(s) and not necessarily those of the NHS, the NIHR or the Department of Health. 


\section{References}

1. Morse, D., R.C. Dailey, and J. Bunn, Prehistoric multiple myeloma. Bulletin of the New York Academy of Medicine, 1974. 50(4): p. 447-458.

2. Solly, S., Remarks on the pathology of mollities ossium; with cases. Medico-chirurgical transactions, 1844. 27: p. 435-498.8.

3. Macintyre, W., Case of Mollities and Fragilitas Ossium, accompanied with urine strongly charged with animal matter. Medico-chirurgical transactions, 1850. 33: p. 211-232.

4. Bence Jones, H., Papers ON CHEMICAL PATHOLOGY;: Prefaced by the Gulstonian Lectures, read at the Royal College of Physicians, 1846. The Lancet, 1847. 50(1247): p. 88-92.

5. Bladé, J. and L. Rosiñol, Complications of Multiple Myeloma. Hematology/Oncology Clinics of North America, 2007. 21(6): p. 1231-1246.

6. Mundy, G.R., Mechanisms of bone metastasis. Cancer, 1997. 80(8 Suppl): p. 1546-56.

7. Taube, T., et al., Abnormal bone remodelling in patients with myelomatosis and normal biochemical indices of bone resorption. (0902-4441 (Print)).

8. Mundy, G.R., Myeloma bone disease. European Journal of Cancer, 1998. 34(2): p. 246-251.

9. Yoneda, T. and T. Hiraga, Crosstalk between cancer cells and bone microenvironment in bone metastasis. Biochemical and Biophysical Research Communications, 2005. 328(3): p. 679687.

10. Owen, M., Marrow stromal stem cells. J Cell Sci Suppl, 1988. 10: p. 63-76.

11. Zhang, Y., et al., Mechanisms Underlying the Osteo- and Adipo-Differentiation of Human Mesenchymal Stem Cells. ScientificWorldJournal, 2012. 2012.

12. Cawthorn, W.P., et al., Bone marrow adipose tissue is an endocrine organ that contributes to increased circulating adiponectin during caloric restriction. Cell Metab, 2014. 20(2): p. 36875.

13. Herroon, M.K., et al., Bone marrow adipocytes promote tumor growth in bone via FABP4dependent mechanisms. Oncotarget, 2013. 4(11): p. 2108-23.

14. Falank, C., H. Fairfield, and M.R. Reagan, Signaling Interplay between Bone Marrow Adipose Tissue and Multiple Myeloma cells. Frontiers in Endocrinology, 2016. 7: p. 67.

15. Shafat, M.S., et al., Leukemic blasts program bone marrow adipocytes to generate a protumoral microenvironment. Blood, 2017. 129(10): p. 1320-1332.

16. Tabe, Y., et al., Bone Marrow Adipocytes Facilitate Fatty Acid Oxidation Activating AMPK and a Transcriptional Network Supporting Survival of Acute Monocytic Leukemia Cells. Cancer Res, 2017. 77(6): p. 1453-1464.

17. Ricci, C., et al., Normal age-related patterns of cellular and fatty bone marrow distribution in the axial skeleton: MR imaging study. Radiology, 1990. 177(1): p. 83-8.

18. D., W., Magnetic resonance imaging of bone marrow: a review - Part I. Journal of American Osteopathic College of Radiology, 2012. 1(2): p. 2-12.

19. Fazeli, P.K., et al., Marrow fat and bone--new perspectives. J Clin Endocrinol Metab, 2013. 98(3): p. 935-45.

20. Gurevitch, O., S. Slavin, and A.G. Feldman, Conversion of red bone marrow into yellow Cause and mechanisms. Med Hypotheses, 2007. 69(3): p. 531-6.

21. Aunan, J.R., W.C. Cho, and K. Soreide, The Biology of Aging and Cancer: A Brief Overview of Shared and Divergent Molecular Hallmarks. Aging Dis, 2017. 8(5): p. 628-642.

22. Hasegawa, T., et al., The PPARgamma-selective ligand BRL-49653 differentially regulates the fate choices of rat calvaria versus rat bone marrow stromal cell populations. BMC Dev Biol, 2008. 8: p. 71. 
23. Hardaway, A.L., et al., Bone marrow fat: linking adipocyte-induced inflammation with skeletal metastases. Cancer Metastasis Rev, 2014. 33(0): p. 527-43.

24. Lecka-Czernik, B., C.J. Rosen, and M. Kawai, Skeletal aging and the adipocyte program: New insights from an "old" molecule. Cell Cycle, 2010. 9(18): p. 3648-54.

25. Styner, M., et al., Bone marrow fat accumulation accelerated by high fat diet is suppressed by exercise. Bone, 2014. 64: p. 39-46.

26. Caers, J., et al., Neighboring adipocytes participate in the bone marrow microenvironment of multiple myeloma cells. Leukemia, 2007. 21(7): p. 1580-4.

27. Coppack, S.W., Pro-inflammatory cytokines and adipose tissue. Proc Nutr Soc, 2001. 60(3): p. 349-56.

28. Devlin, M.J., Why does starvation make bones fat? Am J Hum Biol, 2011. 23(5): p. 577-85.

29. Hardouin, P., T. Rharass, and S. Lucas, Bone Marrow Adipose Tissue: To Be or Not To Be a Typical Adipose Tissue? Front Endocrinol (Lausanne), 2016. 7: p. 85.

30. Takeshita, S., et al., Age-related marrow adipogenesis is linked to increased expression of RANKL. J Biol Chem, 2014. 289(24): p. 16699-710.

31. Fidler, I.J., The pathogenesis of cancer metastasis: the 'seed and soil' hypothesis revisited. Nat Rev Cancer, 2003. 3(6): p. 453-8.

32. Brown, M.D., et al., Promotion of prostatic metastatic migration towards human bone marrow stoma by Omega 6 and its inhibition by Omega 3 PUFAs. Br J Cancer, 2006. 94(6): p. 842-53.

33. Gazi, E., et al., Direct evidence of lipid translocation between adipocytes and prostate cancer cells with imaging FTIR microspectroscopy. J Lipid Res, 2007. 48(8): p. 1846-56.

34. Hardaway, A.L., et al., Marrow adipocyte-derived CXCL1 and CXCL2 contribute to osteolysis in metastatic prostate cancer. Clin Exp Metastasis, 2015. 32(4): p. 353-68.

35. Templeton, Z.S., et al., Breast Cancer Cell Colonization of the Human Bone Marrow Adipose Tissue Niche. Neoplasia, 2015. 17(12): p. 849-861.

36. Zhou, B.O., et al., Bone marrow adipocytes promote the regeneration of stem cells and haematopoiesis by secreting SCF. Nat Cell Biol, 2017. 19(8): p. 891-903.

37. Boyd, A.L., et al., Acute myeloid leukaemia disrupts endogenous myelo-erythropoiesis by compromising the adipocyte bone marrow niche. Nat Cell Biol, 2017. 19(11): p. 1336-1347.

38. UK, C.R., Myeloma incidence statistics. 2014.

39. Angtuaco, E.J., et al., Multiple myeloma: clinical review and diagnostic imaging. Radiology, 2004. 231(1): p. 11-23.

40. Lauby-Secretan, B., et al., Body Fatness and Cancer - Viewpoint of the IARC Working Group. New England Journal of Medicine, 2016. 375(8): p. 794-798.

41. Sezer, O., et al., Immunocytochemistry reveals RANKL expression of myeloma cells. Blood, 2002. 99(12): p. 4646-7; author reply 4647.

42. Fu, J., et al., Multiple myeloma-derived MMP-13 mediates osteoclast fusogenesis and osteolytic disease. J Clin Invest, 2016. 126(5): p. 1759-72.

43. Colucci, S., et al., Soluble decoy receptor 3 modulates the survival and formation of osteoclasts from multiple myeloma bone disease patients. Leukemia, 2009. 23(11): p. 213946.

44. Qiang, Y.W., et al., Dkk1-induced inhibition of Wht signaling in osteoblast differentiation is an underlying mechanism of bone loss in multiple myeloma. Bone, 2008. 42(4): p. 669-80.

45. Eda, H., et al., Regulation of Sclerostin Expression in Multiple Myeloma by Dkk-1: A Potential Therapeutic Strategy for Myeloma Bone Disease. J Bone Miner Res, 2016. 31(6): p. 1225-34.

46. Trotter, T.N., et al., Adipocyte-Lineage Cells Support Growth and Dissemination of Multiple Myeloma in Bone. (1525-2191 (Electronic)).

47. Zhang, X.G., et al., Interleukin-6 dependence of advanced malignant plasma cell dyscrasias. Cancer, 1992. 69(6): p. 1373-6. 
48. Sprynski, A.C., et al., The role of IGF-1 as a major growth factor for myeloma cell lines and the prognostic relevance of the expression of its receptor. Blood, 2009. 113(19): p. 4614-26.

49. Jourdan, M., et al., Tumor necrosis factor is a survival and proliferation factor for human myeloma cells. Eur Cytokine Netw, 1999. 10(1): p. 65-70.

50. Borset, M., et al., Hepatocyte growth factor and its receptor c-met in multiple myeloma. Blood, 1996. 88(10): p. 3998-4004.

51. Reseland, J.E., et al., Abnormal adipokine levels and leptin-induced changes in gene expression profiles in multiple myeloma. Eur J Haematol, 2009. 83(5): p. 460-70.

52. Liu, Z., et al., Mature adipocytes in bone marrow protect myeloma cells against chemotherapy through autophagy activation. (1949-2553 (Electronic)).

53. Fowler, J.A., et al., Host-derived adiponectin is tumor-suppressive and a novel therapeutic target for multiple myeloma and the associated bone disease. Blood, 2011. 118(22): p. 587282.

54. Dalamaga, M., et al., Low circulating adiponectin and resistin, but not leptin, levels are associated with multiple myeloma risk: a case-control study. (1573-7225 (Electronic)).

55. Venkateshaiah, S.U., et al., NAMPT/PBEF1 enzymatic activity is indispensable for myeloma cell growth and osteoclast activity. Experimental hematology, 2013. 41(6): p. 547-557.e2.

56. Pang, J., et al., Resistin induces multidrug resistance in myeloma by inhibiting cell death and upregulating $A B C$ transporter expression. (1592-8721 (Electronic)).

57. Nieman, K.M., et al., Adipocytes promote ovarian cancer metastasis and provide energy for rapid tumor growth. Nat Med, 2011. 17(11): p. 1498-503.

58. Paccou, J., et al., The Role of Bone Marrow Fat in Skeletal Health: Usefulness and Perspectives for Clinicians. J Clin Endocrinol Metab, 2015. 100(10): p. 3613-21.

59. D'Ippolito, G., et al., Age-related osteogenic potential of mesenchymal stromal stem cells from human vertebral bone marrow. J Bone Miner Res, 1999. 14(7): p. 1115-22.

60. Devlin, M.J. and C.J. Rosen, The bone-fat interface: basic and clinical implications of marrow adiposity. Lancet Diabetes Endocrinol, 2015. 3(2): p. 141-7.

61. Halade, G.V., et al., High Fat Diet-Induced Animal Model of Age-associated Obesity and Osteoporosis. The Journal of nutritional biochemistry, 2010. 21(12): p. 1162-1169.

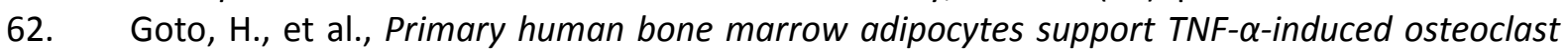
differentiation and function through RANKL expression. Cytokine, 2011. 56(3): p. 662-668.

63. McDonald, M.M., et al., Inhibiting the osteocyte-specific protein sclerostin increases bone mass and fracture resistance in multiple myeloma. Blood, 2017. 129(26): p. 3452-3464.

64. Pozzi, S., et al., In vivo and in vitro effects of a novel anti-Dkk1 neutralizing antibody in multiple myeloma. Bone, 2013. 53(2): p. 487-96. 